\title{
Considerations for assessing frail older adults requesting medical assistance in dying
}

\author{
Sarah Engelhart MD, Nathan M. Stall MD, Kieran L. Quinn MD PhD
}

— Cite as: CMAJ 2022 January 17;194:E51-3. doi: 10.1503/cmaj.210729

I n 2016, medical assistance in dying (MAiD) was decriminalized in Canada with the passage of Bill C-14. Under Bill C-14, competent adults (aged $\geq 18 \mathrm{yr}$ ) with a serious and incurable condition in an advanced state of irreversible decline who were experiencing intolerable physical or psychological suffering and whose death was reasonably foreseeable could be eligible for MAiD. ${ }^{1}$ After its passage, legal challenges were brought before the Superior Court of Quebec, who ruled that the original legislation was too restrictive and in violation of the Canadian Charter of Rights and Freedoms. ${ }^{2}$ This led to the passage of Bill C-7 in March 2021, which amended Canada's criminal code to remove "reasonably foreseeable death" as an eligibility criterion, thus creating 2 distinct pathways for MAiD, where death either is or is not reasonably forseeable. ${ }^{3}$ One patient population likely to be affected by these changes is older adults considered to be frail. Clinicians should consider whether factors contributing to frailty are reversible when considering requests for MAiD.

MAiD for those in whom death is not reasonably forseeable is subject to additional safeguards including a minimum 90-day period for assessing eligibility and mandatory assessment by a clinician with expertise in the patient's condition. ${ }^{3}$ Bill C-7 also permits MAiD through an advanced directive if eligibility was determined when the patient was capable, which is relevant for patients with dementia or those at high risk of developing delirium (both of which often co-exist with frailty).

Frailty is a syndrome characterized by reduced physiologic reserve that renders an individual more susceptible to adverse outcomes in response to stressors. ${ }^{4}$ Frailty is an underrecognized clinical entity that is distinct from normal aging. In frailty, an accelerated decline results from an accumulation of health deficits, leading to dysregulation of multiple systems over time. Many interrelated biological (e.g., genetics), clinical (e.g., underlying comorbidities) and socioeconomic (e.g., social isolation) factors contribute to this accelerated decline, with frailty being a final common pathway. ${ }^{4} \mathrm{~A}$ recent qualitative study from the Netherlands reported that, among 53 patients who underwent MAiD for "multiple geriatric syndromes," a combination of medical, social and existential issues was often associated with unbearable suffering leading to the MAiD request in the absence of a life-threatening condition. ${ }^{5}$ When considering MAiD

\section{Key points}

- Recent changes to Canadian legislation effectively created 2 pathways for medical assistance in dying (MAiD), where death is or is not reasonably foreseeable, with implications for frail older adults.

- Although a reasonably foreseeable death is no longer an eligibility criterion for MAiD, it remains an important and difficult determination for MAiD assessors because it has implications on whether patients are subject to additional safeguards.

- Frailty is an underrecognized clinical entity that can be the source of enduring and intolerable suffering for patients who request MAiD.

- MAiD assessments for frail patients are challenging because of the dynamic nature of frailty and ambiguities in the interpretation of the eligibility criteria as they relate to frailty.

- Improved data collection and clinician education about frail patients who request MAiD are needed, along with enhanced integration of clinicians with expertise in frailty into the committees that inform MAiD policy, guidelines and legislation.

for frail individuals, it is essential that clinicians address this complex constellation of contributing factors, some of which may be modifiable. For example, frailty related to social isolation, depression and food insecurity may need to be addressed differently than frailty from poorly controlled diabetes, congestive heart failure and recurrent falls.

Assessments for frail individuals who request MAiD are challenging given ambiguities in the interpretation of whether frailty is irreversible and incurable, and whether it causes intolerable suffering. Frailty is neither a static nor a dichotomous state, but rather a dynamic condition characterized by transitions between varying degrees of severity. Given the many contributing factors that lead to frailty, determining the trajectory of a patient's overall frailty status, amid episodes of decompensation, makes predicting improvement (or lack thereof) in patients with frailty challenging. The new 90-day waiting period for those requesting MAiD in the absence of a reasonably foreseeable death is a safeguard against the possibility of frailty reversal after an episode of acute decompensation. For individuals with mild frailty, 
comprehensive interventions by multidisciplinary teams addressing both medical and social factors may help improve frailty status. ${ }^{6}$ However, when frailty becomes advanced, it is unlikely to improve, even with these interventions, and the overall trajectory is more predictable. ${ }^{4,6-8}$

Frailty can cause intolerable physical, psychological and existential suffering from a loss of function and independence. ${ }^{9} \mathrm{~A}$ systematic review showed that frail individuals experience severe psychological distress, pain and functional limitations at levels comparable to those with other life-limiting conditions such as cancer. ${ }^{9}$ In Canada, routinely collected data characterize the nature of suffering described by people requesting MAiD. Unfortunately, these data are not specifically reported for those requesting MAiD on the basis of frailty. Still, 2019 data on all individuals who received MAiD showed that suffering was most commonly related to the loss of ability to engage in meaningful life activities (82.1\% of cases) and to perform activities of daily living $(78.1 \%$ of cases $)^{10}$ - sources of suffering common to patients with frailty.

To be eligible for MAiD, patients and assessors must agree that "reasonable and available means of alleviating suffering have been seriously considered." Because frailty is a heterogeneous syndrome, the approach to alleviate suffering must be individualized to the constellation of contributing factors for each frail patient. Assessors should also ensure that MAiD requests are voluntary and not the result of external pressure, which is particularly relevant in the potentially vulnerable population of older adults.

Predicting whether death is reasonably foreseeable in patients considered to be frail is also not straightforward. Numerous studies have shown that frailty is associated with shortened survival compared with nonfrail adults. ${ }^{4,8,11,12}$ Important factors that affect prognosis include the severity of frailty, ${ }^{11,12}$ the number of episodes of hospitalization ${ }^{13}$ and the speed of frailty progression. ${ }^{14}$ Two large Canadian studies of community-dwelling adults with severe frailty reported a median survival of 3.5-4 years. ${ }^{11,12}$ The development of acute illness portends a worse prognosis, both by increasing the likelihood of death and reducing the likelihood of future improvements in frailty status among patients who do survive..$^{13,15}$ Determining eligibility for MAiD on the basis of a reasonably foreseeable death may consider a person's future risk of hospitalization that may subsequently lead to worsened disability and dependence. Although studies help clinicians estimate survival for frail patients, there is still a lack of clarity on how to define a reasonably foreseeable death. For instance, 2 independent assessors could conceivably conclude that a median survival of 4 years for a patient with severe frailty either supports or refutes the reasonable foreseeability of death criterion for MAiD. This key determination has the potential to lead clinicians to forgo additional safegaurds or the potential to prolong patient suffering, depending on its outcome.

Health care systems, clinicians and decision-makers must address these challenges through further research, education and policy changes. Health care systems need to understand how MAiD is being used by patients with frailty. Reporting the number of requests for MAiD on the basis of frailty, as is done with other conditions, should be mandatory. ${ }^{10}$ This should include the proportion of frail individuals found eligible, those deemed to have a reasonably foreseeable death, those with other life-limiting diagnoses for whom frailty is the primary cause of suffering and those whose deaths are deemed not reasonably foreseeable who subsequently die while waiting an extended period to receive MAiD.

Continued education about frailty as it relates to diagnosis, prognosis and suffering is vital for all clinicans and may be accomplished through the creation of MAiD clinical practice guidelines. Integrating clinicians with expertise in frailty into committees that create MAiD guidelines, write policy briefs on the interpretation and determination of a reasonably foreseeable death, and work to inform future MAiD legislation, may help ensure that policies and processes are inclusive and applicable to older adults with frailty. Consultation with geriatricians may lend confidence to the complex clinical judgments involved in MAiD assessments. However, some geriatricians and elderly care specialists are conscientious objectors to MAiD. In acting as independent consultants (and not treating physicians), it is important to formulate clear questions regarding the diagnosis and prognosis of frailty, and not necessarily around the patient's overall eligibility for MAiD. Moreover, access to these specialists is rather limited in Canada and may delay the completion of assessments in a timely manner. Some generalists with expertise in the care of older adults (e.g., family physicians, internists) may be able to provide consultation; however, further clarification is needed on what constitutes an "expert" in the condition causing suffering.

The recent widespread adoption of virtual care during the COVID-19 pandemic may offer an innovative opportunity to create a virtual MAiD consultation service to overcome barriers in access to specialists. Difficulties using digital platforms among some older adults and concerns about privacy in conducting these virtual consultations may limit the extent to which virtual care can be used in this context. However, evolving strategies are under development to address these concerns and improve virtual communication in older adults. ${ }^{16}$

The Canadian population continues to age, with increasing rates of medical comorbidity and associated suffering. A predictable rise in requests for MAiD because of frailty can therefore be expected. In turn, important changes are needed to ensure that all people in Canada have fair and equitable access to their desired end-of-life care.

\section{References}

1. Bill C-14. An Act to amend the Criminal Code and to make related amendments to other acts (Medical Assistance in Dying). 1st session, 42nd Parliament, 2016.

2. Nicol J, Tiedemann M. Legislative summary of Bill C-7: An Act to amend the Criminal Code (medical assistance in dying). In: Legal and Social Affairs Division PlaRS, editor. Ottawa: Library of Parliament; 2020.

3. Bill C-7. An Act to amend the Criminal Code (Medical Assistance in Dying). 2nd Session, 43rd Parliament, 2021.

4. Clegg A, Young J, Iliffe S, et al. Frailty in elderly people. Lancet 2013;381:752-62.

5. van den Berg V, van Thiel G, Zomers M, et al. Euthanasia and physicianassisted suicide in patients with multiple geriatric syndromes. JAMA Intern Med 2021;181:245-50. 
6. Beswick AD, Rees K, Dieppe P, et al. Complex interventions to improve physical function and maintain independent living in elderly people: a systematic review and meta-analysis. Lancet 2008;371:725-35.

7. Gill TM, Gahbauer EA, Allore HG, et al. Transitions between frailty states among community-living older persons. Arch Intern Med 2006;166:418-23.

8. Mitnitski A, Bao L, Rockwood K. Going from bad to worse: a stochastic model of transitions in deficit accumulation, in relation to mortality. Mech Ageing Dev 2006;127:490-3.

9. Stow D, Spiers G, Matthews FE, et al. What is the evidence that people with frailty have needs for palliative care at the end of life? A systematic review and narrative synthesis. Palliat Med 2019;33:399-414.

10. First annual report on Medical Assistance in Dying in Canada, 2019. Ottawa: Health Canada; 2020.

11. Rockwood K, Song X, Mitnitski A. Changes in relative fitness and frailty across the adult lifespan: evidence from the Canadian National Population Health Survey. CMAJ 2011;183:E487-94.

12. Rockwood K, Song X, MacKnight C, et al. A global clinical measure of fitness and frailty in elderly people. CMAJ 2005;173:489-95.

13. Brummel NE, Bell SP, Girard TD, et al. Frailty and subsequent disability and mortality among patients with critical illness. Am J Respir Crit Care Med 2017;196:64-72.

14. Stow D, Matthews FE, Hanratty B. Frailty trajectories to identify end of life: a longitudinal population-based study. BMC Med 2018;16:171.

15. Gill TM, Gahbauer EA, Han L, et al. The relationship between intervening hospitalizations and transitions between frailty states. J Gerontol A Biol Sci Med Sci 2011;66:1238-43.

16. Nieman $\mathrm{CL}$, Oh ES. Connecting with older adults via telemedicine. Ann Intern Med 2020;173:831-2.

Competing interests: Nathan Stall is the 2022 Ontario Liberal Party candidate for Toronto - St. Paul's. Kieran Quinn serves as a volunteer member of the clinical advisory council for the Ontario Palliative Care Network. No other competing interests were declared.

This article has been peer reviewed.

Affiliations: Department of Medicine (Engelhart, Stall, Quinn), University of Toronto; Division of Internal Medicine and Geriatrics (Stall, Quinn), Sinai Health System; Division of Palliative Care (Quinn), Sinai Health System, Toronto, Ont.

Contributors: All authors contributed to the conception, writing and revision of this manuscript. All authors approved the final version for publication.

Content licence: This is an Open Access article distributed in accordance with the terms of the Creative Commons Attribution (CC BYNC-ND 4.0) licence, which permits use, distribution and reproduction in any medium, provided that the original publication is properly cited, the use is noncommercial (i.e., research or educational use), and no modifications or adaptations are made. See: https://creativecommons.org/licenses/by-nc-nd/4.0/

Disclaimer: Nathan Stall is an associate editor for CMAJ and was not involved in the editorial decision-making process for this article.

Correspondence to: Kieran Quinn, kieran.quinn@mail.utoronto.ca 